\title{
Clinical application of urine antigen detection in early onset group B streptococcal disease
}

\author{
E D G McIntosh, H E Jeffery
}

\begin{abstract}
The aim of this study was to test the sensitivity and specificity of antigen detection for group B streptococcus (GBS) from the urine of neonates with early onset GBS sepsis. GBS sepsis was defined as early $(<48$ hours) signs of sepsis in a neonate colonised with GBS. Neonates of 26 weeks' gestation or more, considered at risk for sepsis, were prospectively investigated for one year. Investigations included culture of superficial swabs to assess colonisation, blood culture, and the Wellcogen Strep B latex particle agglutination test on urine. Of 188 neonates investigated, $17(9 \%)$ had GBS sepsis. The urine antigen test had a sensitivity of $88 \%$ and specificity of $98 \%$. The positive predictive value was $79 \%$ and the negative predictive value $99 \%$. Blood culture was positive in only five neonates $(29 \%)$. The annual incidence of GBS sepsis was 4.0 per 1000 and of blood culture positive GBS disease was 1.2 per 1000 live births. Three neonates died. The application of the urine antigen test of clinical neonatal practice is discussed.
\end{abstract}

\section{(Arch Dis Child 1992;67:1198-200)}

The currently accepted definition of early onset group B streptococcal disease is of a baby with clinical signs of infection, usually respiratory distress and a positive blood and/or cerebrospinal fluid (CSF) culture. However, Webber et al have described babies with early onset pneumonia caused by group B streptococcus (GBS) of whom only $46 \%$ had positive blood cultures. ${ }^{1}$ The remaining $54 \%$ were heavily colonised with GBS. Their study did not evaluate urinary GBS antigen detection. Webber's study suggests that a definition requiring positive blood and/or CSF culture may underestimate the incidence of GBS sepsis.

The detection of GBS antigen by methods such as the latex particle agglutination (LPA) test have been studied. The theoretical advantages of this test, compared with traditional blood culture, include antigen detection in body fluids in concentrations as low as $60-70 \mathrm{ng} / \mathrm{ml},{ }^{2}$ the detection of antigen even if prior antibiotic therapy has resulted in minimal live or even dead organisms, and the ability to test sequential urine specimens. Furthermore, once urine has been collected the test takes only 20 minutes to perform.

We have used antigen detection by the LPA method in babies with suspected sepsis and have examined the sensitivity and specificity of urine antigen detection for babies with positive blood culture, and for babies with a clinical diagnosis of GBS sepsis based on clinical signs and colonisation with GBS.

\section{Methods}

Neonates of 26 weeks' gestation or more considered at risk for sepsis entered the study prospectively for one year from November 1986 to October 1987 inclusive. They were considered at risk if one or more of the following criteria were fulfilled: positive antenatal genital swab for GBS, spontaneous onset of preterm labour, prolonged rupture of membranes ( $>12$ hours), intrapartum fever $\left(>37.5^{\circ} \mathrm{C}\right)$, fetal distress, birth asphyxia and/or any of the following occurring within 48 hours of birth-unexplained respiratory distress, sustained tachycardia $(>160$ beats $/ \mathrm{min}$ ), shock, temperature $<36.5^{\circ} \mathrm{C}$ or $>37 \cdot 5^{\circ} \mathrm{C}$. Microscopy and culture of ear and umbilical swabs and gastric aspirate, blood culture, and the Wellcogen Strep B LPA test were performed when indicated as soon after birth as practicable.

The Wellcogen Strep B LPA test is designed to detect polysaccharide wall antigen released from the GBS into various body fluids (for the purpose of this study urine was tested by bag collection after thorough cleansing of the skin). The test was used according to the manufacturer's instructions. That is, the urine was boiled for a minimum of five minutes, centrifuged for 10 minutes at $3000 \mathrm{rpm}$ and one drop of supernatant tested against a control latex and one drop against a test latex. Agglutination, if present, was recorded after gently rocking the test card for three minutes.

In the neonates at risk of sepsis, early onset GBS disease was defined as either clinical signs (unexplained respiratory distress, sustained tachycardia $>160$ beats/min, shock, temperature $<36.5^{\circ} \mathrm{C}$ or $>37.5^{\circ} \mathrm{C}$ within 48 hours of birth) in a baby colonised by GBS, or a positive blood culture before the onset of clinical signs (performed because of one or more of the antenatal risk factors). The absence of GBS disease was defined as either clinical signs in a noncolonised baby or no signs in a colonised baby.

From the at risk group of infants a subgroup of 100 neonates without disease were randomly selected in order to establish (a) the proportion of antenatal compared with postnatal risk factors and (b) the significance of the neutrophil count and placental histology.

The sensitivity, specificity, and positive and negative predictive values of the Wellcogen Strep B LPA test were calculated by applying the 
test results to the above definition of early onset GBS disease. The $95 \%$ confidence limits were calculated for the sensitivity and the specificity of the LPA test. Fisher's exact and $\chi^{2}$ tests were used in the analysis of risk factors. The study was carried out at a time before the routine or widespread use of antenatal genital swabs for the detection of GBS and before the routine or widespread use of antibiotics in mothers (or babies) where antenatal risk factors for GBS infection were present. Nevertheless, some women were swabbed if there was prolonged rupture of the membranes $(>12$ hours) at term, preterm rupture of membranes, or intrapartum fever.

\section{Results}

There were 4282 live births during the one year study period. One hundred and eighty eight neonates with antenatal or postnatal risk factors for early infection were screened for sepsis using all three of the following tests: blood culture; surface swabs and/or gastric aspirate microscopy and culture; and urinary LPA test. Of the 17 neonates who had GBS disease, 16 were colonised with GBS and had clinical signs, and one was asymptomatic with a positive blood culture. The remaining 171 neonates did not have GBS disease.

The sensitivity, specificity, and positive and negative predictive values for the LPA test are shown in table 1. There were four false positive tests. Two occurred in babies colonised with GBS, one baby's mother had received intrapartum antibiotics, and the fourth was neither colonised nor had the mother received antibiotics. Five of the 17 neonates with GBS disease $(29 \%$, $95 \%$ confidence interval $7 \%$ to $51 \%$ ) had blood cultures positive for GBS. One of these had no clinical signs of sepsis. All five neonates had a positive LPA test on urine. There were two false negative tests, both in neonates with clinical signs of sepsis and colonised with GBS. The mother of one of these babies had received intrapartum antibiotics. A repeat urine test was not performed in either baby.

Using the traditional definition of GBS sepsis, a positive blood (or CSF) culture in a neonate with clinical signs or one with maternal risk factors, the sensitivity of the LPA test is $5 / 5$ $(100 \%)$ and the specificity is $169 / 183(92 \%)$.

Of the 12 neonates with positive LPA tests but negative blood cultures, 11 had an antenatal risk factor (including nine whose mothers had intrapartum fever $>37 \cdot 5^{\circ} \mathrm{C}$ ).

Table 2 shows the number of neonates with and without early onset GBS disease, and the proportion that were preterm, neutropenic

Table 1 Sensitivity and specificity of the LPA test

\begin{tabular}{cccc}
\hline & $\begin{array}{l}\text { GBS } \\
\text { disease }\end{array}$ & $\begin{array}{l}\text { No GBS } \\
\text { disease }\end{array}$ & Total \\
\hline $\begin{array}{c}\text { LPA test } \\
\text { positive }\end{array}$ & 15 & 4 & 19 \\
$\begin{array}{c}\text { LPA test } \\
\text { negative }\end{array}$ & 2 & 167 & 169 \\
Total & 17 & 171 & 188 \\
\hline
\end{tabular}

Sensitivity, $88 \%$ (95\% confidence interval $73 \%$ to $100 \%$ ); specificity, $98 \%$ (95\% confidence interval $96 \%$ to $100 \%$ ); positive predictive value, $79 \%$; negative predictive value, $99 \%$.
Table 2 Early onset GBS disease: gestation, neutropenia and chorioamnionitis/vasculitis. Results are number (\%)

\begin{tabular}{lll}
\hline & GBS disease & No GBS disease \\
\hline Preterm & $10 / 17(59 \%)$ & $52 / 100(52 \%)^{*}$ \\
Neutropenia & $4 / 17(24 \%)$ & $8 / 98(8 \%)^{*}$ \\
$\begin{array}{l}\text { Chorioamnionitis or } \\
\text { Vasculitis }\end{array}$ & $9 / 14(64 \%)$ & $34 / 72(47 \%)^{*}$ \\
\end{tabular}

*No significant difference between disease and no disease groups.

according to the criteria of Manroe et $a l,^{3}$ and had placental chorioamnionitis or vasculitis as defined by Russell. ${ }^{4}$ There was no statistical difference between the disease and the non-disease groups for preterm birth, neutropenia or for chorioamniotis/vasculitis, although the apparent trend was for babies with disease to have neutropenia.

The annual incidence of early onset GBS disease was four per 1000 live births. The annual incidence of blood or CSF culture positive GBS disease was 1.2 per 1000 live births. Three preterm neonates died as a result of the disease giving a case fatality rate of $17 \cdot 7 \%$.

\section{Discussion}

This study defined early onset GBS disease as the presence of clinical signs within 48 hours of birth in a neonate colonised by GBS, or positive blood or CSF culture. This definition is somewhat controversial. However, support for its validity is provided by our finding that 11 of 12 neonates with urinary GBS antigen detected in the face of negative blood cultures had recognised maternal risk factors for GBS sepsis. In order to detect GBS disease the urinary LPA test was applied to neonates who had either antenatal or postnatal risk factors for infection. The sensitivity of $88 \%$ and specificity of $98 \%$ for the LPA test in this study suggest a rapid means for presumptively diagnosing early onset GBS disease before formal cultures are available.

Previous studies have examined the sensitivity and specificity of the LPA test in detecting GBS sepsis. $^{2}{ }^{5-11}$ Most have relied on positivity of blood or cerebrospinal fluid culture in order to define the sensitivity and specificity of the LPA test. The present study suggests blood culture is only $29 \%(5 / 17)$ sensitive ( $95 \%$ confidence interval $7 \%$ to $51 \%$ ) in detecting early onset GBS disease.

The lack of specificity when judged by blood culture, creating false positive urinary LPA tests, has been addressed by several authors. Harris et al investigated neonates with suspected sepsis who had positive LPA tests and negative blood cultures. ${ }^{12}$ These authors found that local contamination of the perirectal skin or urinary tract with B streptococcus was an unlikely source of false positive LPA reactions. They concluded that maternal antibiotic pretreatment during labour may represent an important cause of apparent false positive LPA reactions. False positive urine tests have been observed rarely in patients infected with other bacterial pathogens, for example Proteus mirabilis. ${ }^{7}$

Sanchez et al suggested that contamination of bag specimens of urine with GBS from heavy 
perineal and rectal colonisation may produce a positive urine LPA test in an infant with no systemic signs of infection. ${ }^{13}$ Microbiological tests carried out at the Royal Prince Alfred Hospital, Sydney, suggested that heavy contamination with $10^{7}$ organisms $/ \mathrm{ml}$ or more of GBS was required to cause a 'false positive' LPA test (Dr R Benn, personal communication).

Ascher et al believe that the meaning of a positive urine antigen result with a concomitant negative blood culture remains unresolved, but suggest that gastric absorption of GBS antigen may play a part. ${ }^{14}$

As the negative predictive value of the LPA test is $99 \%$, a negative result in an at risk infant who is asymptomatic can support a clinical decision not to treat with antibiotics. This presumes GBS disease is a leading cause of bacterial morbidity in the particular neonatal unit. While early antibiotic treatment is indicated in the neonate when there are clinical signs of sepsis, the decision to cease antibiotics is usually made on the basis of rapid clinical improvement in the absence of positive cultures. A negative LPA test provides additional evidence with which safely to terminate antibiotic treatment.

The annual incidence of early onset GBS disease in this study was 4 per 1000 live births, a rate that is within the reported range in the literature but is higher than recent reports for the USA and Europe. ${ }^{15}$ This figure was threefold greater than the annual incidence of 1.2 per 1000 live births as determined by blood culture over the same period of time in the present study. The definition of early onset GBS disease used in this study may result in overdiagnosis. It is, however, clinically safer than relying on blood culture which underestimates the disease at least twofold. ${ }^{1}$

The implications of using neonatal blood culture alone to estimate early onset GBS disease have both collective and individual maternal relevance. Collectively, strategies for prevention of disease in any given maternity unit will need to assess both the maternal carriage rate and the incidence of the disease in the neonate. The effectiveness of any intervention, such as intrapartum chemoprophylaxis, will rely on evidence of a reduction in neonatal disease incidence as one important outcome. A test with $29 \%$ sensitivity as found for blood culture in this study is inadequate to define either the need for intervention or the effect of intervention. Alternatively, the LPA test applied to urine is easy to implement and acceptable with a sensitivity of $88 \%$.

For an individual mother, detection of early onset GBS disease has important implications, not only for appropriate and immediate treatment of her neonate but also for her future pregnancies. The likelihood that inadequate maternal antibody levels to GBS contribute to neonatal susceptibility to GBS disease and the reported recurrence in subsequent pregnancies suggests an increased risk for perinatal disease in future pregnancies. ${ }^{16}$

In conclusion, this study suggests that detection of GBS antigen in the urine by the LPA test is valuable in improving clinical neonatal practice. This is based on the evaluation of the test when applied to a clinical definition of early onset GBS disease. The sensitivity of $88 \%$ and specificity of $98 \%$ is within acceptable limits. The $99 \%$ negative predictive value is a valuable adjunct to safely limiting unnecessary antibiotic use. The increased rate of disease detection when the test was compared with traditional blood culture, has implications for appropriate antibiotic treatment of the individual neonate, as well as future siblings. This test also has a role in defining neonatal GBS disease more accurately when evaluating preventive strategies.

We wish to thank Dr Richard Benn, head of the department of microbiology, Royal Prince Alfred Hospital for his support and advice throughout the study.

EDGM was supported by Smith Kline Beecham.

1 Webber S, Wilkinson AR, Lindsell D, Hope PL, Dobson SRM, Isaacs D. Neonatal pneumonia. Arch Dis Child 1990;65:207-11.

2 Baker CJ, Rench MA. Commerical latex agglutination for detection of group B streptococcal antigen in body fluids f Pediatr 1983;102:393-

3 Manroe BL, Weinberg AG, Rosenfeld CR, Browne R. The neonatal blood count in health and disease. I. Reference values for neutrophil cells. $\mathcal{F}$ Pediatr 1979;95:89-98.

4 Russell P. Inflammatory lesions of the human placenta. I American fournal of Diagnostic Gynecology and Obstetric 1979;1:127-37.

5 Bromberger PI, Chandler B, Gezon H, Haddow JE. Rapid detection of neonatal group B streptococcal infections by latex agglutination. F Pediatr 1980;96:104-6.

6 Webb BJ, Baker CJ. Commercial latex agglutination test for rapid diagnosis of group B streptococcal infection in infants. $\mathcal{f}$ Clin Microbiol 1980;12:442-4.

7 Ingram DL, Suggs DM, Pearson AW. Detection of group $B$ streptococcal antigen in early-onset and late-onset group B streptococcal disease with the Wellcogen Strep B group B streptococcal disease with the Wellcogen Strep

8 Hamoudi AC, Marcom MJ, Cannon HJ, McCleod RE Hamoudi AC, Marcom MJ, Cannon HJ, McCleod RE. the diagnosis of group B streptococcal sepsis in neonates. Pediatr Infect Dis 1983;2:432-5.

9 Friedman CA, Wender DF, Rawson JE. Rapid diagnosis of group B streptococcus infection ultilizing a commercially available latex agglutination assay. Pediatrics 1984 73:27-30.

10 Rabalais GP, Bronfin DR, Daum RS. Evaluation of a commercially available latex agglutination test for rapid diagnosis of group B streptococcal infection. Pediatr Infect Dis f 1987;6:177-81.

11 Blecker DL, Zimbo MJ, Erbe MB, Mortensen JE, Dickinson B. Polyclonal antigroup B streptococcus latex antigen detection test. Pediatr Infect Dis $\mathcal{F}$ 1989;8:251-2.

12 antigen detection test. Pediatr Infect Dis f 1989;8:251-2. Investigation of apparent false-positive urine latex particle Investigation of apparent false-positive urine latex particle agglutination tests for the detection of group

13 Sanchez PJ, Siegel JD, Cushion NB, Threlkeld N. Significance of a positive urine group B streptococca latex agglutination test in neonates. F Pediatr 1990; 116 601-6.

14 Ascher DR, Wilson S, Mendiola J, Fisher GW. Group B streptococcal latex agglutination testing in neonates. $\mathcal{J}$ Pediatr 1991;119:458-61.

15 Mayon-White RT. The incidence of GBS disease in neonates in different countries. Antibiot Chemother 1985;35:17-27

16 Baker CJ, Edwards MS. Group B Streptococcal infections In: Remington JS, Kelin JO, eds. Infectious diseases of the fetus and newborn infant. 3rd Ed. Philadelphia: WB Saunders, 1990:742-811. 